Culture et histoire dans l'espace roman

\title{
Non siamo i primi
}

\section{Božidar Stanišić}

\section{OpenEdition}

\section{Journals}

\section{Edizione digitale}

URL: https://journals.openedition.org/cher/11176

DOI: 10.4000/cher.11176

ISSN: 2803-5992

\section{Editore}

Presses universitaires de Strasbourg

\section{Edizione cartacea}

Data di pubblicazione: 30 juin 2013

Paginazione: 93-102

ISBN: 978-2-35410-054-4

ISSN: 1968-035X

\section{Notizia bibliografica digitale}

Božidar Stanišić, «Non siamo i primi», reCHERches [Online], 10 | 2013, online dal 08 février 2022, consultato il 10 février 2022. URL: http://journals.openedition.org/cher/11176 ; DOI: https://doi.org/ 10.4000/cher.11176

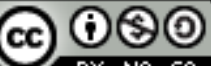

Ce(tte) œuvre est mise à disposition selon les termes de la Licence Creative Commons Attribution Pas d'Utilisation Commerciale - Partage dans les Mêmes Conditions 4.0 International. 


\title{
Non siamo i primi
}

\author{
BožIDAR StanIšIĆ
}

Scrittore

\begin{abstract}
$Q$
uest'autunno, alla stazione di Strasburgo, aspettando il pullman per Francoforte, avevo scritto una lettera a un vecchio amico poeta che, abitando in Bosnia, gode letteralmente di tutti i vantaggi sia della sua dissidenza che dell'emarginazione da parte delle cricche culturali, in primis letterarie e giornalistiche. Nella lettera a lui indirizzata, scritta a mano (quasi da un dinosauro all'altro) al tavolo di un bar della stazione, avevo descritto il mio viaggio da Udine al capoluogo alsaziano e le ragioni della partecipazione al convegno sul tema Scrivere altrove. Il destinatario è già da tempo interessato al fenomeno degli stranieri che scrivono anche o solo in italiano. Così gli avevo descritto le ragioni per le quali ero andato proprio a Strasburgo e perché, ormai da anni, grazie al mio rifiuto di essere considerato scrittore migrante, sono ignorato dagli accademici-sacerdoti e dai vari organizzatori di festival, convegni e incontri letterari sulla letteratura migrante. Lui mi conosce: nell'ex-Jugoslavia non ero della razza degli autoinvitati, né lo sono - dico - in Italia. (Poiché al mio vecchio amico piace leggere sulle città che non aveva visitato e che non visiterà, gli avevo descritto l'umidità del vento autunnale dell'Alsazia, i rintocchi dell'orologio della Cattedrale, i profumi dei crauti nei ristorantini...).

Quella lettera all'amico, però, non è stata del tutto altruista. È servita anche a me, soprattutto per raccogliere le parole espresse nella mia relazione e sintetizzarle. A pochi giorni dalla mia partenza per Strasburgo, dal Comitato editoriale di «El Ghibli», in occasione del ventennio della letteratura d'immigrazione o letteratura nascente in Italia, anch'io ho ricevuto la loro lettera, a cui, ecco, rispondo. Cosciente della complessità delle domande
\end{abstract}


e della necessità che su questi argomenti si debba sentire una moltitudine di voci, aggiungo solo che, a mio avviso, della nominazione proposta venti anni fa è rimasto poco, essenzialmente forse nulla. Lo affermo sperando che il dibattito sulla tematica toccherà anche altre questioni nel contesto dei temi letterari non estranei alla letteratura, dalle quali spuntano pure la problematica delle cosiddette seconde generazioni e della multiculturalità. Lo sottolineo per nulla casualmente, perché mi chiedo se dopo la schedatura socio-culturale delle seconde generazioni, fra una decina d'anni non sarà sorta la problematica delle terze, quarte, quinte... generazioni. In realtà, questo mio discorso è dedicato a loro sperando che lotteranno molto meglio di noi, sia immigrati che scrittori immigrati di oggi, per la dignità della scrittura e l'uguaglianza delle persone, immigrate e non. Certo, anche questa speranza ha un peso, come ha l'interrogativo sulla multiculturalità senza osmosi più complesse fra le culture attualmente presenti in Italia e in Europa. Poi mi chiedo pure quanto i figli si interrogheranno sulla sostanza della immigritudine ${ }^{1}$ dei loro genitori, ovvero quanta ce n'era nella loro coscienza sociale e culturale e quale relazione avranno con essa.

Ora, passati parecchi giorni dal Convegno, a volte mi pento perché, anche se brevemente, mi ero auto-presentato in modo ridondante: nato in Bosnia, ho vissuto nel mio paese fino all'estate del 1992 lavorando come insegnante di lettere e scrivendo sulla critica letteraria e le belle arti, testi radiofonici, libri per l'infanzia; prima dello scoppio della guerra civile nell'ex-Jugoslavia ero attivo come presidente di uno dei rari movimenti per la pace in Bosnia; appena incominciata la guerra sono fuggito assieme a mia moglie e mio figlio; dal 1992 vivo in Italia, in un paesello vicino a Udine, a $40 \mathrm{~km}$ dal confine italo-sloveno; sono uno dei cinque milioni di persone che, dai territori dell'ex-Jugoslavia frantumata, fra il 1991 e il 2000, essendosi trovati indesiderati, terrorizzati o insicuri nella psicosi dei risvegliati nazionalismi nelle nuove patrie (in un istante per loro diventate sbagliate), hanno dovuto cambiare indirizzo. Mi chiedo se avessi dovuto dire, invece, soltanto questo: sono uno scrittore, scrivo altrove dall'estate del '92 e forse, per far piacere a più di qualcuno, avrei dovuto aggiungere la parola migrante? (Essendo severo con me stesso, soprattutto nei momenti in cui per qualche vis maior abbandono il mio credo alla necessità della continuità della scrittura - nulla dies sine linea, dubito spesso se sono davvero uno scrittore oppure solo un

1 Questo termine l'ho inventato in riferimento a quello del poeta A. Césaire, che più di mezzo secolo fa pronunciò il termine negritudine, volendo sintetizzare la coscienza di essere neri, con la loro storia e identità. 
autore di alcune delle opere narrative, poetiche, saggistiche e teatrali finora realizzate e pubblicate.) Però già da tempo non dubito più se sono uno scrittore migrante. Semplicemente, non mi sento uno scrittore migrante. Scrittore forse, ma migrante no. Se ancora in certe occasioni sono considerato e, ahimè, trattato come tale - osservo con il sorriso di chi ha capito che stare quasi in una minoranza assoluta a volte provoca pure l'impotenza mentale di opporsi sia a sottotitoli del genere che a contenitori di moda.

A questo punto vorrei spiegare in breve il perché del mio rifiuto. Sì, è vero che una quindicina di anni fa ero molto entusiasta perché qualcosa si era mosso nel campo culturale e letterario italiano per dare voce agli autori stranieri, di cui la netta maggioranza sono extracomunitari ${ }^{2}$. Non voglio presentarmi in modo immodesto, $\mathrm{ma}$ in italiano avevo già pubblicato alcuni libri (si tratta delle traduzioni di miei racconti e poesie scritte in serbocroato) e molti articoli e saggi scritti in italiano, corretti da alcuni amici. Perciò con il cuore aperto, a partire dalla metà degli anni Novanta, avevo accettato volentieri di essere presente in alcune antologie e panoramiche poetiche e narrative della letteratura d'immigrazione nonché a partecipare ad alcuni concorsi letterari, fra i quali si distingueva quello dell'Associazione EksఓTra.

Passati alcuni anni, grazie soprattutto al contatto diretto con il soggetto nominato per ultimo, a me aveva incominciato a rivelarsi il fatto che la letteratura d'immigrazione - e non solo secondo i responsabili di quest'associazione - sarebbe dovuta restare nel suo guscio, e ciò per molti motivi. (A questo punto mi fermo, disponibile ad ogni dialogo.) Aggiungo solo che questo concorso annuale, partito anche per valorizzare la produzione narrativa degli stranieri che scrivono in italiano e quindi nato da un'idea molto valida, anzi nobile, con il passare degli anni, a mio avviso, è degenerato, servendo ormai quasi soltanto all'autopromozione della già nominata associazione. C’è qualche prezzo da pagare per questi miei atteggiamenti che sembrano radicali, non solo con l'Eks\&Tra, ma con molti organizzatori - sia del passato, che attuali - di incontri sulla letteratura d'immigrazione? Ecco, non volendo cadere nella trappola di racconti provinciali, dico subito: oltre agli organizzatori degli incontri trentini «Il gioco degli specchi», l'associazione «La tenda» di Milano, le riviste on-line «El Ghibli» e «Sagarana», alcuni amici scrittori e poeti stranieri e soprattutto

2 Nella patria di Dante la burocrazia politica non era e non è molto delicata, perciò questo termine ormai non può venir rimosso neppure da qualche tsunami di vera cultura d'accoglienza. 
italiani che comprendono la mia opposizione in una luce diversa dai sacerdoti sulla letteratura d'immigrazione - c'era e c'è poca attenzione sulla mia possibile presenza ai vari dibattiti, conferenze e serate letterarie ispirate da questa tematica. Pare sia molto bello non sentire voci critiche, né opinioni diverse!

Meglio, però soli, o quasi soli, che in qualche cassetto. Se dovessi scegliere tra l'essere considerato uno scrittore migrante o un uccello migratore, accetterei più volentieri quest'ultima considerazione. Almeno si potrebbe volare sopra mari e terre lontani.

In realtà, come si sentono gli oggetti chiusi in un cassetto? Non ho una risposta perché non conosco il loro linguaggio. Perciò credo sia meglio interrogarsi su come si sente un essere umano chiuso in una cella carceraria. Innocente o colpevole che sia, sappiamo tutti che non si sente bene. Quando in Italia osservo il fenomeno della letteratura scritta dagli immigrati, un particolare non mi sfugge: avendo accettato l'etichetta di scrittori migranti, ovvero ascrivibili alla categoria della letteratura d'immigrazione prodotta in lingua italiana, ritengo che la netta maggioranza degli autori stranieri abbia rinnegato il senso della chiusura in un cassetto o in un carcere. Detto in altri termini, dentro si sta meglio, si è più sicuri e, infine, si evita di porre alcuni interrogativi sostanziali perché inquietanti, di cui almeno uno potrebbe risultare scomodo.

Io, scrivendo e pubblicando anche in italiano, posso considerarmi uno scrittore italiano e appartenere alla letteratura italiana contemporanea? Sulla nostra appartenenza, di noi che scriviamo altrove, si sa, spesso decidono altri. Più di uno, in tutti questi lunghi anni, si è interessato su chi sono e su come mi sento: scrittore ex jugoslavo, serbo, serbo bosniaco, bosniacoerzegovese o italiano. Da tempo rispondo che mi sento davvero uno scrittore di nessuno. E aggiungo che scrivo sia in un mio yiddish (è il mio vissuto della lingua serbo-croata, ormai ufficialmente inesistente), che in italiano.

È semplice comprendere il perché di nessuno? Dirò qualcosa in proposito. Ecco un esempio: circa dieci anni fa, a Trieste, venne pubblicata una panoramica sul vissuto letterario di questa città nella letteratura serba. Anche se avevo scritto più di qualcosa sul tema, nessun frammento delle mie opere è stato inserito in questa panoramica. Poi, forse, ho finalmente capito che il titolo della panoramica narrativa, Il dizionario di un paese che scompare ${ }^{3}$, nella quale sono presente con un mio racconto, era più che

3 AA.VV., Il dizionario di un paese che scompare, a cura di N. Janigro, Roma, Ed. Il Manifesto, 1994. 
profetico. E non solo nei miei riguardi, ma in quelli di decine di scrittori che hanno lasciato la nostra ex patria. E molti per sempre. Che aggiungere? Che esistiamo noi, scrittori della cosiddetta diaspora, e loro, che sono rimasti nella cosiddetta patria. Eppure tutto ciò fa parte dello scrivere altrove, inclusa questa divisione che pare netta: noi (fuggiti, partiti) e loro (rimasti), che riecheggia, in qualche modo, in Italia e altrove: noi, scrittori di altre parti del mondo che abbiamo scelto di scrivere in italiano oppure anche in italiano, e loro, i veri autoctoni.

Vi sembra che io mi stia lamentando? E che lo faccia pure quando provo a definire dove, in quale spazio inserire gli scrittori o gli autori della narrativa o della poesia in lingua italiana, quindi anche me stesso? Voglio illustrare ciò in modo semplice: non credo sia giusto, e poi sarebbe noioso, elencare quali lavori umili ho fatto in tutti questi anni del mio lungo, a volte strano, soggiorno in Italia. Come me, lo facevano pure molti altri (anche se non tutti...). Accenno solo al fatto che da anni sono attivo anche nel campo delle mediazioni linguistiche e culturali, soprattutto nella regione Friuli-Venezia Giulia nella quale vivo. Lo faccio nelle scuole, negli ospedali, nei centri d'igiene mentale... E pure presso alcuni comuni. Né io, né i miei colleghi abbiamo avuto, né abbiamo, un ufficio. A volte neppure un tavolino e una sedia negli atri e nelle sale d'attesa. Però siamo sempre tra e con gli immigrati. E paragonando questa situazione (diciamo determinata dalla tolleranza dei soggetti che chiedono i nostri interventi - anche se non so che cosa ci sia da tollerare, il verbo in sé e per sé non è bello, né molto chiaro), con quella della letteratura d'immigrazione, mi pare che essa sia rimasta in sala d'attesa. È vista da tutti coloro che entrano, quindi sia dagli italiani (cioè dagli autoctoni), che dagli immigrati stessi. Ma da chi deve essere ricevuta, chi deve controllare la sua tessera? Ma tutto ciò, in confronto alla necessità di fare letteratura sia in lingua italiana che nella lingua madre degli immigrati, credo sia irrilevante. Non c'è nulla di più importante dell'opera stessa. Il resto, i riconoscimenti, i premi, le pacche sulle spalle dei buonisti o i rifiuti dei ciechi e sordi xenofobi o di xenofobi principianti, gira attorno come una nuvola di moscerini.

Avrei, però, una domanda semplice: «Siamo i primi?». E anche oggi contraddico sia chi ama restare dentro quel cassetto, attraverso il quale si va a partecipare a festival, convegni, conferenze e, a volte, si ricevono anche riconoscimenti, sia i sacerdoti delle accademie: No, non siamo i primi.

A questo punto dovrebbero spuntare dal passato i destini di personaggi illustri come Conrad, Nabokov, Kundera, Marai, i due Milosz, Brodskij, 
Rushdie... e non solo, ma molti altri, sin dai secoli lontani, diciamo da Ovidio, fino ai giorni nostri. Semplicemente, siamo con loro sulla stessa verticale della Storia. E con i loro nomi, destini e opere si rivela anche l'interrogativo a quali letterature appartengono (apparteniamo). Non credo che si tratti di un argomento diverso dal nostro, perché di conseguenza sorge un'inevitabile domanda: «la vera patria degli scrittori (non solo migranti) è la lingua che usano, oppure quella nella quale sono nati e partiti, negli ultimi trent'anni, verso l'Italia e altrove, spesso con un biglietto di sola andata, come direbbe Erri De Luca?» Solo andata, fatto che condiziona molti in maniera permanente. Uno scrivere altrove - è forse un'altra interpretazione, sicuramente più onesta e pertinente, sulla condizione di essere scrittori con il permesso di soggiorno in Italia. Scrivere altrove, dovrebbe essere l'argomento più importante sul fenomeno della nostra e altrui presenza, cioè degli altri scrittori non nati con la camicia linguistica.

Ammetto: non sono innocente (forse il mio risveglio di fronte al fenomeno della cosiddetta letteratura d'immigrazione è stato tardivo?), ma - ripeto - non mi piacciono né quel cassetto, né quella cella già nominata. Quindi aggiungo: molti anni fa, anch'io stavo, direi volontariamente, dentro quel cassetto. Ma ne sono davvero uscito? Formalmente credo di no. Sono presente in vari volumi antologici e panoramici sia di prosa che di poesia, in varie presentazioni ecc., tutte pubblicate in Italia, con il sottotitolo di letteratura d'immigrazione. E non mi vedo dentro quella cella: non solo perché scrivo prevalentemente nella mia lingua madre, il serbo-croato (frantumata in quattro standard abbastanza ripuliti: bosniaco, croato, montegrino e serbo), perciò soggettivamente nominato il mio yiddish. Scrivo in italiano quando sento dentro di me una profonda necessità di esprimere qualcosa proprio in questa lingua, insistendo fra me e me sulla sincerità di questa scelta, spesso ascoltando, come se mi fossero sussurrate, le parole di Vera Linhartova: «Lo scrittore non è prigioniero di una sola lingua. Egli, infatti, prima di essere uno scrittore è un uomo libero». Ma non dimentico nemmeno quelle di Danilo Kiš: "Convinciti che la lingua in cui scrivi è la migliore di tutte, poiché non ne hai un'altra. Convinciti che la lingua in cui scrivi è la peggiore di tutte, anche se non la cambieresti con nessuna».

(Una parentesi: credo che, in relazione alla libertà e alla lingua, spunti un interrogativo semplice, forse anche doloroso. Perché siamo così pochi, noi che abbiamo mantenuto l'abitudine e la volontà di scrivere nella lingua madre? So che non sono molti che, come me, hanno avuto l'occasione veramente straordinaria di avere vicino un traduttore come lo è Alice 
Parmeggiani, a volte Ljiljana Avirovic, non solo traduttrici, ma pure ottime conoscitrici della letteratura degli slavi del sud. Oltre a ciò sarebbe interessante chiedersi perché anche autori stranieri provenienti, ad esempio, da zone francofone, slavofone, ispanofone e germanofone, hanno deciso di scrivere solo in italiano?).

Ritengo sia giunto il momento di dire che in nessun modo intendo svalutare le ricerche su questo fenomeno letterario. Ho avuto il piacere di conoscere persone serie, umane, profonde, fra cui il professor Armando Gnisci, Mia Lecomte, Cristina Mauceri e Raffaele Taddeo. Ma devo tornare sull'argomento: chi tra gli autori e gli scrittori stranieri in Italia (e altrove) ancora immagina la letteratura d'immigrazione come un ombrello di protezione, perché non lo apre davvero? Non lo fa perché si bagnerà? In tutti questi anni si sono affermati autori validi, con delle opere rispettabili. Però, osservando il fenomeno della nascita e dello sviluppo della letteratura d'immigrazione, non riesco a non tastare, almeno in qualche modo, il polso alla società alla quale ci rivolgiamo con la nostra scrittura, e a non interrogarmi su come siamo accolti e chi siamo, sia per i lettori italiani che per gli immigrati stessi.

Neppure questo lo faccio per lamentarmi.

Se dico ad alcuni amici, oppure mentre sto svolgendo delle mediazioni culturali o delle lezioni sulla letteratura nelle scuole italiane, che vale la pena leggere, ad esempio, le poesie di Gëzim Hajdari, mi chiedono chi sia costui. Rispondo: è un poeta italiano contemporaneo. Ma come Gëzim, e poi Ajdari? Sì, dico, Hajdari è (anche) un poeta italiano perché scrive in italiano. E l'imbarazzo dei miei interlocutori non viene scalfito. Neppure se raccomando la narrativa di Julio Monteiro Martins o di Pap Khouma. Ahimè, Kh...Kh...! E si ripete lo stesso filo di domande e risposte. In qualche modo se la cava Laila Wadia. Diciamo, a metà: ormai c'è qualche Laila fra le ragazze giovani, però non la salva quella $\mathrm{W}$ del suo cognome... Peccato, poteva passare per italiana, ma non ci è riuscita. E così via (per non parlare del mio nome). Però, in alcune occasioni, gli studenti delle superiori e delle università non ci vedono nulla di anormale in questo acquisto della lingua italiana da parte di poeti e narratori immigrati.

Ripeto che è chiaro che sulla determinazione della nostra appartenenza, quella di chi scrive altrove, in realtà decidono altri, che pur portano il loro bagaglio culturale. Lo portiamo tutti, insieme al peso o alla leggerezza della nostra condizione umana, irripetibile perciò drammaticamente bella. In quel bagaglio ci sono pure dei meccanismi di rifiuto, di classificazione, di 
accettazione, di sentirsi superiori o inferiori, di capire o non capire l'altro e il diverso. Un domani non solo i critici o gli esperti in materia diranno che siamo questo o quello, oppure una terza cosa. Ma, soggettivamente, non è ciò che mi interessa molto. É un momento per esprimermi sullo scrivere altrove, forse l'unico vero argomento degno della nostra attenzione.

Dov'è il mio altrove? (Proverò a sintetizzarlo, sperando di non essere noioso ripetendo la descrizione e la definizione delle mie finestre).

La mia casa a Zugliano, paesello alle porte di Udine, ha le sue finestre orientali e quelle occidentali. Dalle prime vedo le vette delle montagne in Slovenia. Nei giorni tersi riesco a vedere le cime del Tricorno, a sua volta la montagna più alta della ex Jugoslavia. Il paesello in cui abito è sì vicinissimo a Udine, ma è pur vicino a Gorizia e a Trieste (per la quale, negli anni dopo la seconda guerra mondiale, il mio barbiere d'infanzia fece cinque anni di servizio militare). Per molti occidentali ancor oggi sono i punti d'inizio dell'Europa orientale. Le mie finestre occidentali mi offrono la vista sui tetti del Centro di accoglienza per immigrati stranieri, profughi e richiedenti asilo "Ernesto Balducci" dove, all'inizio della mia permanenza in Italia, ero ospitato assieme alla mia famiglia. Per me è diventato già da tempo la metafora del ritorno alla vita e alla scrittura. Quando ero, come molti esuli dell'ex-Jugoslavia, forse non più di un numero, là ho ritrovato non solo accoglienza, ma anche sentimenti di amicizia e di solidarietà. E più lontano, a una quarantina di chilometri, si vedono le cime di Piancavallo. Ai piedi della montagna si trova Aviano, cittadina che ospita la base Usaf, luogo da cui, con la scusa di guerre umanitarie o di operazioni antiterrorismo, decollavano e decollano i cacciabombardieri. Pare che io sia uno dei testimoni di questo confine invisibile fra due scelte della nostra Storia in Movimento: della pace, della solidarietà e del dialogo da una parte, della prepotenza e della chiusura verso ogni possibile interlocuzione dall'altra.

Il mio orizzonte, in questa estrema periferia del Nord-Est d'Italia, è realmente cambiato? Essenzialmente credo di no. Anni fa, in Bosnia, vivevo fra gente di diverse culture, costumi, religioni. Voglio ribadirlo anche in quest'occasione: mai immaginavo di dover vivere in un altro paese. Con gli anni ho sempre meno dubbi se sia davvero del tutto normale vivere laddove non siamo nati. Se immagino di nuovo le finestre della mia casa natia, anch'esse erano orientali e occidentali. Le prime mi offrivano uno sguardo su tutto ciò che ci ha lasciato in eredità l'Impero ottomano durante i lunghi secoli d'occupazione, le seconde verso l'Europa alla quale, credo, apparteniamo con tutte le specificità della nostra storia e della nostra cultura. 
(Abituato a sentire, e non solo in Italia, che la Bosnia è la porta d'Oriente, a volte chiedo ai miei interlocutori se sono passati, almeno una volta, per una porta. Rimangono sorpresi e tuttavia rispondono di sì. Allora, dico, è chiaro che la porta d'Oriente dall'altro lato è anche la porta d'Occidente?). E di nuovo vivo fra gente di varie nazionalità, religioni, lingue, costumi... Vivo pure dentro i fenomeni di accoglienza dell'altro e del diverso da una parte, e il rifiuto dall'altra. Sì, è una realtà che, anche per me, è più fantastica di tutto ciò che dovrebbe risultare fantastico nel suo senso più stretto. E non volendo osservare nulla in bianco e nero, neppure sui fenomeni di xenofobia e di razzismo fra gli immigrati stessi, scrivo non dimenticando le ragioni per cui sono stato costretto a non vivere più né in Bosnia, né in un altro Paese sorto dalla frantumazione della Jugoslavia.

E, vi ripeto - non mi lamento, neppure quando mi considerano uno scrittore-ponte, alludendo ai contenuti della mia scrittura. No, non mi oppongo a questa determinazione. Al di fuori dell'eccellente visione dei ponti di Ivo Andrić, il ponte per me è - lo direbbe pure l'amico Massimo Rizzante - un altrove assoluto: "colui che si trova su un ponte è in nessun luogo, ha la sensazione di non avere radici». Anche questo fa parte intensamente dello scrivere altrove, quasi negando del tutto il lato negativo dell'esilio. Rizzante ci riporta alle parole di Josif Brodskij, pronunciate nella conferenza del 1987. In effetti, in Una condizione chiamata esilio, il poeta russo ironizzava sul fatto che lo scrittore in esilio è quasi sempre «un essere retrospettivo [...]. Come i falsi profeti di Dante, il nostro uomo ha la testa perpetuamente rivolta all'indietro e le lacrime, o la saliva, gli sgorgano giù fra le scapole». Pare che siamo quindi arrivati al come trasformare la condizione di non appartenenza in una condizione di quotidiana libertà; oppure, secondo Vera Linhartova, andiamo verso un altrove sconosciuto per definizione e aperto a tutte le possibilità. Pure Kundera vede nell'esilio una promessa di libertà.

In tutti questi anni, oltre alla domanda «Migrando, chi non diventa straniero?», spesso mi sono soffermato sulla riflessione di José Ortega y Gasset, espressa nelle Meditazioni del Chisciotte": "io sono io e la mia circostanza e se non salvo questa non salvo neanche me». So benissimo che tutti siamo nati in un determinato tempo e luogo, quindi il nostro io è fissato da circostanze spaziali e temporali, però è la circostanza che non mi offre nessuna pace e mi porta oltre la verità che ogni individuo era, è, e resterà unico. Anche da ciò proviene uno dei miei interrogativi: «Chi siamo

4 J. Ortega y Gasset, Meditazioni del Chisciotte, trad. di B. Arpaia, Napoli, Guida, 1986. (Titolo originale: Meditaciones del Quijote, 1914). 
nelle relazioni con gli altri e i diversi? In queste relazioni possiamo (e come?) umanizzare di più le nostre circostanze e le circostanze altrui?» E da questi interrogativi, anche se sembra di volgarizzare nuovamente il pensiero di Y Gasset, nasce un'altra domanda: "Abbiamo occhi abbastanza interessati per osservare il Sud del mondo dalle finestre della Casa Occidentale dove abitiamo, senza importanza sul fatto se siamo immigrati o autoctoni?»

Alla fine mi chiedo se in questo mio sguardo risuoni abbondantemente il significato della parola libertà. Se risuonava, credo lo facesse serenamente. Forse è così perché lo scrivere altrove ci rivela il fatto che la scrittura è limitata solo se noi vogliamo limitarla, dimenticando che non siamo i primi, e che la condizione di scrittore esule o immigrato ha fortemente segnato la letteratura del secolo scorso e continua a farlo anche all'inizio del ventunesimo. E tutto ciò ritengo che succeda al di là dell'illibertà offertaci dalle catalogazioni già osservate, dai concorsi letterari con temi forzati, dagli editori ancora dubbiosi, fino a sentirsi scrittori di nessuno lasciando la risposta vera, quella sulle nostre opere e sul nostro grado di maturità di pensiero, all'unico popolo senza bandiera, quello dei lettori.

P.S. Alcuni giorni fa ho ricevuto la risposta del mio amico, poeta della Bosnia: "Caro amico, mio uccello migratore, capisco i tuoi venti alsaziani, la tua resistenza alla catalogazione. Si, la gente ha bisogno (o soltanto le pare), di classificare, evidenziare, contare, catalogare tutto e tutti... e non è disturbata dal fatto che la questione sia della natura che appartiene agli spazi spirituali, per i quali sinora non è stato costruito nessun catalogo. Non è stato costruito, ma a molti piace costruirlo. Poi, agli scrittori accadono varie cose. Non credo che esistano convegni su medici, pittori o musicisti migranti. Ma a ciò non bisogna dare peso. Anche gli scrittori, fra loro, introducono alcune regole, alcuni segni attraverso i quali distinguersi dagli scrittori del passato, a volte anche dai futuri (a ciò contano superficialmente). Una volta Ivo Andrić, a proposito di un'analisi letteraria sulla sua opera considerata seria e profonda, disse che non aveva capito nulla. Credo sia stato molto sincero. E, per non annoiarti, finisco con il convincimento che una critica possa essere criticata, un'analisi analizzata, ma una poesia o un racconto possano essere scritti una volta sola. È bello ricevere una lettera da paesi lontani, da un migrante o non, che trova una ragione per ricordare un amico.

Il tuo P. 